\section{Comment on "Magnetic-Field-Tuned Quantum Phase Transition in the Insulating Regime of Ultrathin Amorphous Bi Films"}

A recent Letter by Lin and Goldman 11 presented experimental data for the relative magnetoresistance (MR) in disordered thin films, which were interpreted as evidence of a quantum phase transition. Such films are known to exhibit a superconductor (SC)-insulator transition as a function of disorder [2, and a huge peak in the resistance $R(B)$ with magnetic field $B[3,4$. These highly disordered samples were insulating at zero $B$. The experimental results supporting the quantum phase transition scenario are: (a) the relative magnetoresistance, $M R\left(B, B_{0}\right)=\left[R(B)-R\left(B_{0}\right)\right] / R\left(B_{0}\right)$, at $B_{0}=0$ was temperature $(T)$ independent at a specific, non-universal, field $B_{\mathrm{C}}$, and (b) near this point all the different- $T$ curves collapsed upon rescaling $R=R_{\mathrm{C}} F\left(\left|B-B_{\mathrm{C}}\right| / T^{1 / \nu z}\right)$, where $\nu$ and $z$ were interpreted as the critical exponents of the transition. In this comment we present an alternative interpretation based on activated transport in a disordered landscape. We first present numerical simulations, and then support them by simple analytic arguments.

Our numerical simulations were performed using a new $a b$ initio technique, based on the disordered negative- $U$ Hubbard model, that fully captures the effects of thermal phase fluctuations [5]. The results of this method describe the observed phenomenology of transport through thin disordered SC films, including the origin of the magnetoresistance peak [6]. Here we report results for more disordered systems, which, as in the experiment, are resistive at zero $B$ (we used an onsite energy standard deviation of $W=6 t$, where $t$ is the lattice hopping integral, onsite interaction $U=1.6 t$, and 0.37 filling). The inset of Fig. 1(b) depicts $R(B)$ for several temperatures, with the resulting MR shown in Fig. 1(a), where the main experimental result is reproduced - following a peak, the MR isotherms cross at a constant magnetic field. Near that point, all the curves collapse (Fig. 1b), using the same scaling analysis as in [1, with $\nu z=0.89$. The sample displays no notable phenomenon in the local currents and chemical potential at $B_{\mathrm{C}}$.

Since our numerical calculations neglect quantum fluctuations, the source of our crossing point $B_{\mathrm{C}}$ cannot be the putative quantum phase transition 11. To understand the crossing we note that both in the theory and in the experiment, the resistance is activated, $R(B, T)=R_{0}(B) \mathrm{e}^{T_{\mathrm{A}}(B) / T}$, with $T_{\mathrm{A}}(B)$ the activation temperature at field $B$, and $R_{0}(B) \approx h / 4 e^{2}$ is the high temperature resistance. Fig. 1 (a) shows that $T_{\mathrm{A}}(B)$, in agreement with experiment, is a non-monotonic function, and, in fact, $B_{\mathrm{C}}$ corresponds to $T_{\mathrm{A}}\left(B_{\mathrm{C}}\right)=T_{\mathrm{A}}(0)$. If $R(B, T)$ obeys the activated behavior above, $M R(B, 0)$ becomes $T$-independent at $B=B_{\mathrm{C}}$. Moreover, expanding $T_{\mathrm{A}}(B)$ around $B=B_{\mathrm{C}}$, we find that the scaling function

$$
\operatorname{MR}\left(B, B_{0}\right)=\frac{R_{0}(B)}{R_{0}\left(B_{0}\right)}\left(1+\frac{T_{\mathrm{A}}^{\prime}\left(B_{\mathrm{C}}\right)\left(B-B_{\mathrm{C}}\right)}{T}\right)-1,
$$

is in agreement with the experimental fitted form with $\nu z=1$. (The deviations from perfect scaling come from the weak dependence of $R_{0}(B)$ on $B$, and from the deviations, both experimentally and numerically, from simple activation at lower temperatures.)

If our interpretation is correct, and $B_{\mathrm{C}}$ was only determined by $T_{\mathrm{A}}\left(B_{\mathrm{C}}\right)=T_{\mathrm{A}}(0)$, the same behavior should be observed in less disordered samples for $M R\left(B, B_{0}\right)$, where $T_{\mathrm{A}}\left(B_{\mathrm{C}}\right)=T_{\mathrm{A}}\left(B_{0}\right)$ and $B_{0}>0$. Indeed in Fig. 1 $(\mathrm{c}, \mathrm{d})$ we present results for a sample with lower disorder $W=t$ that is $\mathrm{SC}$ at $B=0$. Again the MR isotherms all cross at $B=B_{\mathrm{C}}$, with a reasonable collapse. Moreover the inset of Fig. 1(d) depicts the excellent collapse of the experimental data published in Ref. 3] for a lower disorder sample, with $B_{0}=4 \mathrm{~T}$ and $B_{\mathrm{C}}=12.8 \mathrm{~T}$, supporting our scenario.

In summary, using $a b$ initio simulations and analytic arguments, we have demonstrated an alternative explanation of the experimental results of Ref. [1]. The crossing of the MR curves can be understood entirely in terms of activated transport, which our previous analysis attributed to transport through Coulomb blockade islands 6]. Finally, we have made a specific prediction to test our analysis.

G.J. Conduit and Y. Meir

Ben Gurion University, Beer Sheva 84105, Israel

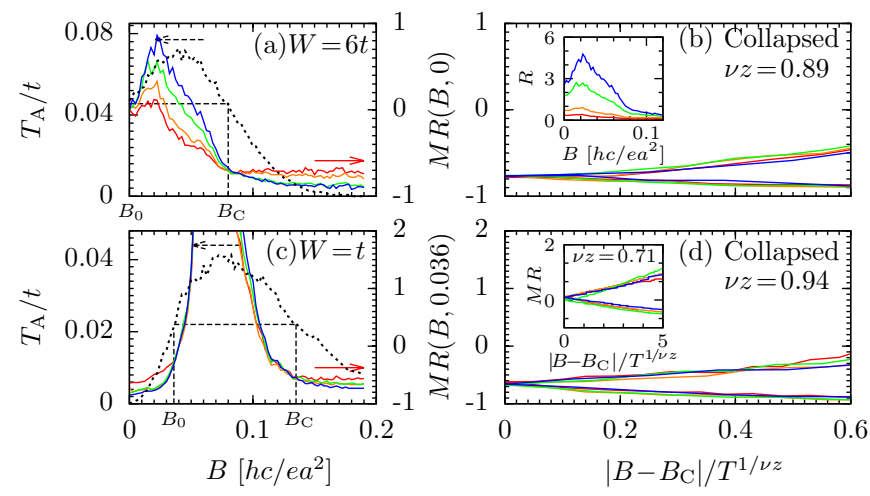

FIG. 1: (Color online) (a,c) The MR curves (solid) and activation temperature (dotted) with magnetic field. The upper plots were taken at disorder $W=6 t$ and the lower at $W=t$. The blue curve (lowest at large fields) is at low temperature $T=0.02 t$, and red high temperature $T=0.07 t$. (b,d) The $\mathrm{MR}$ with scaled magnetic field. The inset (b) shows the variation of resistance with magnetic field and (d) the MR for the experimental data from Ref.[3].
[1] Y.-H. Lin and A.M. Goldman, Phys. Rev. Lett. 106, 127003 (2011).
[2] For a review, see, e.g., A. Goldman, Physics Today, Nov. 
1998, p.41.

[3] G. Sambandamurthy, L.W. Engel, A. Johansson, and D. Shahar, Phys. Rev. Lett. 92, 107005 (2004)

[4] M.A. Steiner, G. Boebinger, and A. Kapitulnik, Phys. Rev. Lett. 94, 107008(2005); H.Q. Nguyen, S.M. Hollen, M.D. Stewart, Jr., J. Shainline, Aijun Yin, J.M. Xu, and
J.M. Valles, Jr., Phys. Rev. Lett. 103, 157001 (2009).

[5] G.J. Conduit and Y. Meir, Phys. Rev. B 84, 064513 (2011)

[6] G.J. Conduit and Y. Meir, arxiv/1111.2941. 